\title{
Use of linear mixed models for genetic evaluation of gestation length and birth weight allowing for heavy-tailed residual effects
}

\author{
Kadir Kizilkaya ${ }^{1,2^{*}}$, Dorian J Garrick ${ }^{1,3}$, Rohan L Fernando ${ }^{1}$, Burcu Mestav², Mehmet A Yildiz ${ }^{4}$
}

\begin{abstract}
Background: The distribution of residual effects in linear mixed models in animal breeding applications is typically assumed normal, which makes inferences vulnerable to outlier observations. In order to mute the impact of outliers, one option is to fit models with residuals having a heavy-tailed distribution. Here, a Student's-t model was considered for the distribution of the residuals with the degrees of freedom treated as unknown. Bayesian inference was used to investigate a bivariate Student's-t (BSt) model using Markov chain Monte Carlo methods in a simulation study and analysing field data for gestation length and birth weight permitted to study the practical implications of fitting heavy-tailed distributions for residuals in linear mixed models.
\end{abstract}

Methods: In the simulation study, bivariate residuals were generated using Student's-t distribution with 4 or 12 degrees of freedom, or a normal distribution. Sire models with bivariate Student's-t or normal residuals were fitted to each simulated dataset using a hierarchical Bayesian approach. For the field data, consisting of gestation length and birth weight records on 7,883 Italian Piemontese cattle, a sire-maternal grandsire model including fixed effects of sex-age of dam and uncorrelated random herd-year-season effects were fitted using a hierarchical Bayesian approach. Residuals were defined to follow bivariate normal or Student's-t distributions with unknown degrees of freedom.

Results: Posterior mean estimates of degrees of freedom parameters seemed to be accurate and unbiased in the simulation study. Estimates of sire and herd variances were similar, if not identical, across fitted models. In the field data, there was strong support based on predictive log-likelihood values for the Student's-t error model. Most of the posterior density for degrees of freedom was below 4. Posterior means of direct and maternal heritabilities for birth weight were smaller in the Student's- $t$ model than those in the normal model. Re-rankings of sires were observed between heavy-tailed and normal models.

Conclusions: Reliable estimates of degrees of freedom were obtained in all simulated heavy-tailed and normal datasets. The predictive log-likelihood was able to distinguish the correct model among the models fitted to heavy-tailed datasets. There was no disadvantage of fitting a heavy-tailed model when the true model was normal. Predictive log-likelihood values indicated that heavy-tailed models with low degrees of freedom values fitted gestation length and birth weight data better than a model with normally distributed residuals.

Heavy-tailed and normal models resulted in different estimates of direct and maternal heritabilities, and different sire rankings. Heavy-tailed models may be more appropriate for reliable estimation of genetic parameters from field data.

\footnotetext{
* Correspondence: kadirk@iastate.edu

${ }^{1}$ Department of Animal Science, lowa State University, Ames, IA 50011 USA
} 


\section{Background}

Animal breeding applications commonly involve the fitting of linear mixed models in order to estimate genetic and phenotypic variation or to predict the genetic merit of selection candidates. Measurement errors and other sources of random non-genetic variation comprise the residual term, the effects of which are often assumed to be normally distributed with zero mean and common variance. These assumptions may make inferences vulnerable to the presence of outliers $[1,2]$. Heavy-tailed densities (such as Student's- $t$ distribution) are viable alternatives to the normal distribution, and provide robustness against unusual or outlying observations when used to model the densities of residual effects. In the event that the degrees of freedom are estimated to be large, i.e. in excess of 30 , these methods converge to normally distributed residuals [3].

Mixed effects linear models with Student's- $t$ distributed error effects have been applied to mute the impact of residual outliers, for example in a situation where preferential treatment of some individuals was suspected [4]. Von Rohr and Hoeschele [5] have demonstrated the application of a Student's- $t$ sampling model under four different error distributions in statistical mapping of quantitative trait loci (QTL). They have determined that additive and dominance QTL and residual variance estimates are much closer to the simulated true values when the data itself is heavy-tailed and the analysis is performed with the skewed Student's- $t$ model rather than with a normal model. Rosa et al. [6] have analyzed birth weight in a reproductive toxicology study and compared normal as well as robust mixed linear models based on Student's- $t$ distribution, Slash or contaminated normal error distributions. Marginal posterior densities of degrees of freedom for the Student's- $t$ and Slash error distributions are concentrated about single digit values, suggesting the inadequacy of the normal distribution for modelling residual effects. The heavy-tailed distributions result in significantly better fit than a normal distribution. Kizilkaya et al. [3] have applied threshold models with normal or Student's- $t$ link functions for the genetic analysis of calving ease scores and they have shown that predictive log-likelihoods strongly favour a Student's- $t$ model with low degrees of freedom in comparison with a normal distribution. Cardoso et al. [7] have used heavy-tailed distributions to study residual heteroskedasticity in beef cattle and have found that a Student's- $t$ model significantly improves predictive loglikelihood value. Chang et al. [8] have compared multivariate heavy-tailed and probit threshold models in the analysis of clinical mastitis in first lactation cows, and have shown that a model comparison strongly supports the multivariate Slash and Student's- $t$ models with low degrees of freedom over the probit model. The objectives of this research were to 1 ) examine by simulation if Bayesian inference under a bivariate Student's- $t$ distribution of residuals can accommodate models with either light-tailed or heavy-tailed residuals, and 2) investigate the practical implications of fitting a Student's- $t$ distribution with unknown degrees of freedom for the residuals in bivariate field data. In both cases, results were compared to those from the conventional approach of assuming bivariate normal (BN) residuals.

\section{Methods}

We first present the theory and methods for multiple traits that are applicable to both the simulation and the analysis of field data on gestation length and birth weight using a model that accommodates heavy-tailed residuals.

\section{Statistical model}

A linear mixed model for animal $i$ is

$$
\mathbf{y}_{i}=\mathbf{X}_{i} \mathbf{b}+\mathbf{Z}_{i} \mathbf{a}+\mathbf{W}_{i} \mathbf{h}+\epsilon_{i}
$$

where $\mathbf{y}_{i}=\left(y_{i, 1} \ldots y_{i, m}\right)^{\prime}$ is a vector of phenotypic values of animal $i$ for $m$ traits, $\mathbf{b}$ is a vector of fixed effects, $\mathbf{a}$ is a vector of random genetic effects, $\mathbf{h}$ is a vector of uncorrelated random effects such as herd effects, $\mathbf{X}_{i}, \mathbf{Z}_{i}$ and $\mathbf{W}_{i}$, are design matrices for animal $i$, corresponding to the vectors of the fixed effects $(\mathbf{b})$, random genetic effects (a), and uncorrelated random effects (h).

Conventional analyses might assume the vector $\epsilon_{i}$ in equation (1) is multivariate normally distributed $(N(\mathbf{0}$, $\left.\mathbf{R}_{0}\right)$ ), where

$$
\mathbf{R}_{0}=\left(\begin{array}{lll}
\sigma_{e_{1}}^{2} & \cdots & \sigma_{e_{1} e_{m}} \\
\vdots & \ddots & \vdots \\
\sigma_{e_{m} e_{1}} & \cdots & \sigma_{e_{m}}^{2}
\end{array}\right) .
$$

In contrast, we assume $\epsilon_{i}$ in (1) is multivariate heavyor light-tailed by expressing the residual in the usual manner but divided by a scalar random variable that varies for each animal $i$ but is consistent across the traits. That is,

$$
\left(\begin{array}{c}
\epsilon_{1} \\
\vdots \\
\epsilon_{m}
\end{array}\right)_{i}=\left(\begin{array}{c}
e_{1} \\
\vdots \\
e_{m}
\end{array}\right)_{i} \lambda_{i}^{-\frac{1}{2}}=\mathbf{e}_{i} \lambda_{i}^{-\frac{1}{2}}
$$

where $\lambda_{i}$ in equation (2) is a positive random variable [9]. Values of $\lambda_{i}$ approaching 0 produce heavy-tailed residuals for both traits, whereas values exceeding 1 
would produce light-tails. The marginal density of $\epsilon_{i}$ is a multivariate Student's- $t$ density with scale parameter $\mathbf{R}_{\mathbf{0}}$ and $\mathrm{df} v$, such that the marginal residual variance becomes $\operatorname{Var}\left(\boldsymbol{\epsilon}_{i} \mid \mathbf{R}_{0}, v\right)=\mathbf{R}_{E}=\mathbf{R}_{0}\left(\frac{v}{v-2}\right)[4,7,9]$.

\section{Prior and full conditional posterior distributions}

A flat prior was assumed for the fixed effects (b). Genetic effects (a) were assumed to be distributed as multivariate normal, with null mean vector and (co)variance matrix $A \otimes \mathbf{G}_{\mathbf{0}}$ where $\mathbf{A}$ is the numerator relationship matrix and $\otimes$ denotes the Kronecker product [10]. Uncorrelated random effects and residuals were assumed to follow multivariate normal distributions with null means and (co)variance matrices $\mathbf{I} \otimes \mathbf{H}_{\mathbf{0}}$ and $\mathbf{I}$ $\otimes \mathbf{R}_{\mathbf{0}}$ where $\mathbf{I}$ is the identity matrix. Flat prior distributions were assigned to $\mathbf{G}_{\mathbf{0}}, \mathbf{H}_{\mathbf{0}}$ and $\mathbf{R}_{\mathbf{0}}$.

The multivariate normal distribution requires no distributional specification of $\lambda_{i}$ in equation (2), because $\lambda_{i}$ $=1$ for all $i=1,2, \ldots, n$. The distribution of $\lambda_{i}$ in equation (2) for multivariate Student's- $t$ is a $\operatorname{Gamma}(v / 2, v / 2)$ distribution with density function

$$
p\left(\lambda_{i} \mid v\right)=\frac{(v / 2)^{v / 2}}{\Gamma(v / 2)} \lambda_{i}^{(v / 2)-1} \exp \left(-\frac{v}{2} \lambda_{i}\right)
$$

Where $\lambda_{i}>0, \Gamma($.$) is the standard Gamma function,$ $i=1,2, \ldots, n$ and $v>0$. A prior of $p(v)=\frac{1}{(1+v)^{2}}$ for $v>$ 0 was assigned to $v$ [3].

Inferences on parameters of interest can be made from the posterior distributions constructed using MCMC methods such as Gibbs sampling or MetropolisHastings [11-13]. The fully conditional posterior distributions of each of the unknown parameters are used to generate proposal samples from the target distribution (the joint posterior). The fully conditional posterior distributions of fixed (b), genetic (a) and uncorrelated random (h) effects are multivariate normal with mean $[\hat{\mathbf{b}}, \hat{\mathbf{a}}, \hat{\mathbf{h}}]$ and covariance matrix $\mathbf{C}$, where $[\hat{\mathbf{b}}, \hat{\mathbf{a}}, \hat{\mathbf{h}}]$ are solutions to Henderson's mixed model equations constructed with heterogeneous residual variances, $\mathbf{R}_{0} \lambda_{i}^{-1}$ and $\mathbf{C}$ is the inverse of this mixed-model coefficient matrix [4]. The (co)variance matrices $\mathbf{G}_{\mathbf{0}}, \mathbf{H}_{\mathbf{0}}$ and $\mathbf{R}_{\mathbf{0}}$ have inverse Wishart conditional posterior distributions, which can also be constructed from $[\hat{\mathbf{b}}, \hat{\mathbf{a}}, \hat{\mathbf{h}}, \hat{\lambda}]$ where $\hat{\lambda}$ is solution for $\lambda_{i}$ [9].

The fully conditional posterior distributions of $\lambda_{i}$ for the multivariate Student's- $t$ model is

$$
\operatorname{Gamma}\left(\frac{v+m}{2}, \frac{1}{2}\left[\mathbf{e}^{\prime} \mathbf{R}_{0}^{-1} \mathbf{e}+v\right]\right)
$$

where $\mathbf{e}=\mathbf{y}_{i}-\mathbf{X}_{i} \mathbf{b}-\mathbf{Z}_{i} \mathbf{a}-\mathbf{W}_{i} \mathbf{h}$.
The fully conditional posterior distribution of $\mathrm{df} v$ for the multivariate Student's- $t$ model does not have a standard form, and so a sampling strategy for nonstandard distributions is required. A random-walk MetropolisHastings $(\mathrm{MH})$ algorithm was used to draw samples for $v$ [11]. In the $\mathrm{MH}$ algorithm, a normal density with expectation equal to the parameter value from the previous MCMC cycle was used as the proposal density. The $\mathrm{MH}$ acceptance ratio was tuned to intermediate rates (40-50\%) during the MCMC burn-in period to optimize MCMC mixing [3]. Sampled values of $v<2$ were truncated to 2 so that covariance matrix, $\mathbf{R}_{E}=\mathbf{R}_{0}\left(\frac{v}{v-2}\right)$, for the residuals of (1) is defined.

\section{Simulation study}

A simulation study was carried out to validate Bayesian inference on the bivariate Student's- $t$ models, and assess the ability of model choice criterion (predictive log-likelihood) to correctly choose the model with better fit. For this purpose, the simulation study was undertaken using three sire models to simulate the bivariate data, these models varying in the nature of the simulated residual effects. We refer to the model used to simulate the data as the true model. These three models were the bivariate normal which effectively has infinite $v(\mathrm{BN}-\infty)$ and the bivariate Student's- $t$ model with $v=4$ or 12 (BSt-4, BSt12). Ten replicated data sets were generated for each of the three true models. Phenotypes of 50 progeny from each of 50 unrelated sires for two traits, $\mathbf{y}_{i}=\left(y_{i, 1} y_{i, 2}\right)^{\prime}$ were simulated using equation (1). The vector of fixed effects b only included a gender effect with $b_{1}=(1190)^{\prime}$ for trait 1 and $b_{2}=\left(\begin{array}{ll}38 & 32\end{array}\right)^{\prime}$ for trait 2 . The random genetic effects (a) and uncorrelated random effects (h) included 50 sires and 100 herds, respectively, assuming:

$$
\left(\begin{array}{l}
\mathbf{a} \\
\mathbf{h}
\end{array}\right) \sim N\left(\left[\begin{array}{l}
\mathbf{0} \\
\mathbf{0}
\end{array}\right],\left[\begin{array}{cc}
\mathbf{G}_{0} \otimes \mathbf{I} & \mathbf{0} \\
\mathbf{0} & \mathbf{H}_{0} \otimes \mathbf{I}
\end{array}\right]\right)
$$

where $\mathbf{G}_{0}$ is the sire (co)variance matrix,

$$
\mathbf{G}_{0}=\left(\begin{array}{cc}
\sigma_{s_{1}}^{2} & \sigma_{s_{1} s_{2}} \\
\sigma_{s_{2} s_{1}} & \sigma_{s_{2}}^{2}
\end{array}\right)=\left(\begin{array}{ll}
2.0 & 1.5 \\
1.5 & 4.0
\end{array}\right),
$$

and $\mathbf{H}_{0}$ is the herd (co)variance matrix

$$
\mathbf{H}_{0}=\left(\begin{array}{cc}
\sigma_{h_{1}}^{2} & 0 \\
0 & \sigma_{h_{2}}^{2}
\end{array}\right)=\left(\begin{array}{cc}
1.5 & 0 \\
0 & 6.0
\end{array}\right) .
$$

Residuals were assumed $\mathbf{e}_{i} \sim N\left(\mathbf{0}, \mathbf{R}_{0}\right)$, where

$$
\mathbf{R}_{0}=\left(\begin{array}{ll}
\sigma_{e_{1}}^{2} & \sigma_{e_{1} e_{2}} \\
\sigma_{e_{2} e_{1}} & \sigma_{e_{2}}^{2}
\end{array}\right)=\left(\begin{array}{ll}
15.0 & 4.0 \\
4.0 & 20.0
\end{array}\right) .
$$


Heritabilities of simulated traits were $h_{1}^{2}=0.43$ and $h_{2}^{2}=0.53$, respectively. For each animal $i, \lambda_{i}$ was 1 for $\mathrm{BN}-\infty$ or generated from $\operatorname{Gamma}(v / 2, v / 2)$ for BSt $-v$ with $v=4,12$. Offspring were assigned to herd and gender groups by random sampling from a uniform distribution.

\section{Gestation length and birth weight data}

Gestation length (GL) up until first calving and the resultant calf birth weight (BW) data were recorded on the national population of Italian Piemontese cattle from January 1989 to July 1998 by Associazione Nazionale Allevatori Bovini di Razza Piemontese (ANABORAPI), Strada Trinità 32a, 12061 Carrù, Italy. Only herds represented by at least 100 records over that period were considered in the study [14], providing a total of 7,883 animals from 677 sires and 747 MGS. Table 1 summarizes the statistics for GL and BW. BSt and BN models given in equation (1) were used to analyze GL and BW data. The fixed effects (b) of dam age in months, sex of the calf, and their interaction were considered by combining eight different firstcalf age group classes (20 to 23,23 to 25,25 to 27,27 to 29,29 to 31,31 to 33,33 to 35 , and 35 to 38 months) with sex of calf for a total of 16 nominal age-sex subclasses. A total of 1,186 herd-year-season (HYS) subclasses were created from combinations of herd, year, and two different seasons (from November to April and from May to October) as in Carnier et al. [15] and Kizilkaya et al., [3] and treated as uncorrelated random effects (h) [14]. The range for number of observations in HYS subclasses was between 1 and 33, and average number of records for HYS effect was 7. The random genetic effects (a) included 1,929 sires (s) and MGS (m) from the pedigree file. While the number of observations ranged from 1 to 406 , average observations for each sire in data file was 12 . We also assumed:

$$
\left(\begin{array}{l}
\mathbf{a} \\
\mathbf{h}
\end{array}\right) \sim N\left(\left[\begin{array}{l}
\mathbf{0} \\
\mathbf{0}
\end{array}\right],\left[\begin{array}{cc}
\mathbf{G}_{0} \otimes \mathbf{A} & \mathbf{0} \\
\mathbf{0} & \mathbf{H}_{0} \otimes \mathbf{I}
\end{array}\right]\right)
$$

where $\mathbf{G}_{0}$ is the sire-MGS (co)variance matrix,

$$
\begin{aligned}
& \mathbf{G}_{0}= \\
& \left(\begin{array}{llll}
\sigma_{s_{G L}}^{2} & & & \\
\sigma_{s_{B W} s_{G L}} & \sigma_{s_{B W}}^{2} & & \\
\sigma_{m_{G L} D_{G L}} & \sigma_{m_{G L} s_{B W}} & \sigma_{m_{G L}}^{2} & \\
\sigma_{m_{B W} s_{G L}} & \sigma_{m_{B W} s_{B W}} & \sigma_{m_{B W} m_{G L}} & \sigma_{m_{B W}}^{2}
\end{array}\right)
\end{aligned}
$$

Table 1 Summary statistics for gestation length (GL) and birth weight (BW) in Italian Piemontese cattle

\begin{tabular}{lccccc}
\hline Trait & N & Mean & Minimum & Maximum & SD \\
\hline GL (day) & 7,883 & 290 & 260 & 320 & 8.1 \\
BW (kg) & 7,883 & 39.6 & 22 & 56 & 4.1 \\
\hline
\end{tabular}

and $\mathbf{H}_{0}$ is the HYS (co)variance matrix,

$$
\mathbf{H}_{0}=\left(\begin{array}{cc}
\sigma_{h_{\mathrm{GL}}}^{2} & 0 \\
0 & \sigma_{h_{B W}}^{2}
\end{array}\right) \text {. }
$$

\section{Marginal residual variances, heritabilities and genetic correlations}

Residual scale parameters $\left(\mathbf{R}_{0}\right)$ in heavy-tailed models cannot be directly compared with the residual (co)variance $\left(\mathbf{R}_{0}\right)$ in the normal model, nor used in estimation of heritabilities, residual or phenotypic correlations. The scale parameters must be appropriately transformed into marginal residual (co)variance parameters $\mathbf{R}_{E}=\left(\begin{array}{ll}\sigma_{E_{G L}}^{2} & \sigma_{E_{G L} E_{B W}} \\ \sigma_{E_{B W} E_{G L}} & \sigma_{E_{B W}}^{2}\end{array}\right)$ for BN and BSt models, using $\mathbf{R}_{E}=\mathbf{R}_{0}$ and $\mathbf{R}_{E}=\mathbf{R}_{0}\left(\frac{v}{v-2}\right)$ where $v>2$, respectively, given by Stranden and Gianola [4] and Cardoso et al. [7].

Heritabilities and genetic correlations are of interest from the perspective of direct and maternal effects in an animal model, but the fitted models for GL and BW included genetic effects for sire and MGS, and some fractions of the genetic effects were included in the residual terms. Transformations were applied to convert the sire-MGS parameters and estimates to their animal model equivalent. The additive genetic (co)variance matrix including direct $(D)$ and maternal $(M)$ genetic variances from sire-MGS model was obtained as $\mathbf{G}_{D M}=$ $\mathbf{P G}_{0} \mathbf{P}^{\prime}$ [16] where $\mathbf{G}_{D M}$ is an additive genetic (co)variance matrix,

$$
\begin{aligned}
& \mathbf{G}_{D M}= \\
& \left(\begin{array}{llll}
\sigma_{D_{G L}}^{2} & & & \\
\sigma_{D_{B W} D_{G L}} & \sigma_{D_{B W}}^{2} & & \\
\sigma_{M_{G L} D_{G L}} & \sigma_{M_{G L} D_{B W}} & \sigma_{M_{G L}}^{2} & \\
\sigma_{M_{B W} D_{G L}} & \sigma_{M_{B W} D_{B W}} & \sigma_{M_{B W} M_{G L}} & \sigma_{M_{B W}}^{2}
\end{array}\right)
\end{aligned}
$$

and $\mathbf{P}$ is an appropriate transformation matrix,

$$
\mathbf{P}=\left(\begin{array}{rrrr}
2 & 0 & 0 & 0 \\
0 & 2 & 0 & 0 \\
-2 & 0 & 2 & 0 \\
0 & -2 & 0 & 2
\end{array}\right)
$$

Direct and maternal $\left(h_{G_{i, j}}^{2}\right)$ heritability, and genetic correlation $\left(r_{G_{i, j} G_{k, j}^{\prime}}\right)$ estimates were obtained from estimates of variance and covariance components according to: 


$$
h_{G_{i, j}}^{2}=\frac{\sigma_{G_{i, j}}^{2}}{\sigma_{s_{i, j}}^{2}+2 \sigma_{s_{i, j} m_{i, j}}+\sigma_{m_{i, j}}^{2}+\sigma_{h_{i, j}}^{2}+\sigma_{E_{i, j}}^{2}}
$$

and

$$
r_{G_{i, j} G_{k, j}^{\prime}}=\frac{\sigma_{G_{i, j} G_{k, j}^{\prime}}}{\sqrt{\sigma_{G_{i, j}}^{2} \sigma_{G_{i, j}^{\prime}}^{2}}}
$$

Where $G$ and $G^{\prime}=D$ or $M, i$ and $k$ for the trait of GL or BW and $j$ for the model of BN or BSt.

Kendall rank correlations between posterior means of sire genetic effects obtained from the BN and BSt models were used to compare the ordering of the genetic evaluations of the sires for GL and BW [17]. Comparisons were also made between rank orders of the top 100 selection candidates from 1,929 animals in the pedigree file for the $\mathrm{BN}$ model.

\section{Model comparison}

Model comparisons in the simulation study, and for the analysis of field data, were carried out using predictive log-likelihoods (PLL) from BN and BSt models. The PLL over all observations $(n)$ under Model $M_{k}(k=\mathrm{BN}$ or BSt $)$ was obtained as:

$$
\begin{aligned}
P L L_{k} & =\sum_{i=1}^{n} \log p\left(\mathbf{y}_{i} \mid \mathbf{y}_{(i)}, M_{k}\right) \\
& =\sum_{i=1}^{n} \log \left(\frac{1}{G} \sum_{j=1}^{G} p^{-1}\left(\mathbf{y}_{i} \mid \boldsymbol{\theta}^{(j)}, M_{k}\right)\right)^{-1}
\end{aligned}
$$

where $\left(\frac{1}{G} \sum_{j=1}^{G} p^{-1}\left(\mathbf{y}_{i} \mid \boldsymbol{\theta}^{(j)}, M_{k}\right)\right)^{-1}$ is the harmonic mean of $p^{-1}\left(\mathbf{y}_{i}^{j=1} \mid \boldsymbol{\theta}^{(j)}, M_{k}\right)$ across G MCMC samples [18]. A PLL difference exceeding 2.5 was used as indication of an important difference in model fit, following Raftery [19].

In the simulation study, the impact of alternative models was quantified by computing the correlations $\left(r_{\hat{\mathbf{a}}}, \mathbf{a}\right)$ between the simulated true (a) and predicted $(\hat{\mathbf{a}})$ sire effects in each of the three fitted models. Further, the prediction error variance (PEV) $\mathrm{V}(\mathbf{a}-\hat{\mathbf{a}}))$ of the sire effects was calculated to provide an informative comparative assessment of model prediction performance. Higher correlations and lower prediction error variances will be associated with fitted models that are better at predicting breeding values than models with low correlations and high prediction error variance. Some fitted models might be significantly better than others from a likelihood framework, yet have little impact on selection response if they do not markedly change correlations.
Minimizing the prediction error variances is important when investment decisions depend upon the magnitude of the sire predictions, not just the ranking of the sires.

\section{MCMC implementation}

Graphical inspection (time series traces) of the chains along with Heidelberger and Welch Diagnostic [20] for the Gibbs output using CODA (Convergence Diagnostics and Output Analysis package in R) [21] were used to determine a common length of burn-in period. A burnin period of 50,000 for simulated and field data analysis was defined as the number of cycles discarded at the start of the MCMC chain to ensure sampling from the correct marginal distributions. A further 50,000 post burn-in MCMC cycles in the simulated and field data analysis were generated for each of the BSt and BN models. Every successive post burn-in sample was retained, so that 50,000 samples were used to infer posterior distributions of unknown parameters. Posterior means of the parameters were obtained from their respective marginal posterior densities. Interval estimates were determined as posterior probability intervals (PPI) obtained from the 2.5 and 97.5 percentiles of each posterior density to provide 95\% PPI. The effective number of independent samples (ESS) for each parameter was determined using the initial positive sequence estimator of Geyer [22] as adapted by Sorensen et al. [23].

\section{Results and discussion}

\section{Simulation study}

The predictive log-likelihood values in Table 2 were computed for BSt and BN models fitted to the simulated heavy-tailed and normal datasets. When the true model had residuals with heavy-tails, the fitted models with heavy-tails (BSt) were significantly better than the normal model (BN). When the true model had normally distributed residuals, all the fitted models performed equally well. The difference in PLL between the fitted models with heavy-tails and the normal model was inversely related to the degrees of freedom of the simulated residuals. Note

Table 2 Comparisons of average predictive loglikelihood' (PLL) from ten replicates between bivariate Student's-t (BSt) and normal (BN) fitted models (in column) for different true simulated models (in rows) with varying residual degrees of freedom (DF)

\begin{tabular}{lrr}
\hline & & Fitted \\
\cline { 2 - 3 } True Model & \\
\hline BSt -4 & BSt & BN \\
BSt-12 & $-1,483$ & $-1,988$ \\
BN $-\infty$ & -718 & -754 \\
\end{tabular}

${ }^{1}$ Predictive log-likelihood values were reported after adding 14,000

${ }^{2}$ Used to simulate data

${ }^{3}$ Used in analysis of simulated data 
Table 3 Average posterior inference on degrees of freedom from ten replicates using the bivariate Student's-t (BSt) fitted model

\begin{tabular}{llccr}
\hline & & \multicolumn{3}{c}{ BSt Fitted Model $^{\mathbf{2}}$} \\
\cline { 3 - 5 } True Parameters & True Model $^{\mathbf{1}}$ & $\mathbf{P M} \pm \mathbf{S E}^{\mathbf{3}}$ & $\mathbf{9 5 \%} \mathbf{P P I}^{\mathbf{4}}$ & ESS $^{\mathbf{5}}$ \\
\hline$v=4$ & $\mathrm{BSt}-4$ & $4.1 \pm 0.06$ & {$[3.6,4.6]$} & 1,594 \\
$v=12$ & $\mathrm{BSt}-12$ & $13.3 \pm 1.18$ & {$[9.8,19.1]$} & 294 \\
$v=\infty$ & $\mathrm{BN}-\infty$ & $2377 \pm 654$ & {$[2140,3365]$} & 14 \\
\hline
\end{tabular}

'Used to simulate data

${ }^{2}$ Used in analysis of simulated data

${ }^{3}$ Posterior mean \pm Standard Error

${ }^{4} 95 \%$ equal-tailed posterior probability interval based on the $2.5^{\text {th }}$ and $97.5^{\text {th }}$ percentiles of the posterior density

${ }^{5}$ Effective sample size

that normally distributed residuals can be thought of as having infinite degrees of freedom, and in this case there were no differences between the fitted models.

Inference on $v$ based on BSt model analysis of BSt-4, $\mathrm{BSt}-12$ and $\mathrm{BN}-\infty$ data sets is given in Table 3. Posterior means of $v$ seems sharp and unbiased, and the $95 \%$ posterior probability intervals for $v$ concentrated on low values for BSt-4 and BSt-12 data sets. Conversely, inference on $v$ for $\mathrm{BN}-\infty$ data was larger than 100 , consistent with what was expected, and the $95 \%$ posterior probability interval was wider by concentrating on values higher than 30 , indicating strong evidence of normally distributed data. Furthermore, relatively larger ESS of $v$ were obtained from BSt-4 and BSt-12 data sets when compared with that from $\mathrm{BN}-\infty$ data sets [3], indicating more samples would be needed to attain a minimum of 100 as advocated by Bink et al. [24] and Uimari et al. [25].

Tables 4, 5 and 6 summarize inferences on sire, herd and marginal error variances based on the replicated datasets from the three different populations, comparing BSt and BN fitted models. Large ESS were attained for sire, herd and marginal error variances, indicating stable MCMC inference. The $95 \%$ posterior probability intervals for sire and herd variance components from the three fitted models widely overlapped and included the true parameter values. Furthermore, the posterior means from the three fitted models were almost identical. When the true model was BSt-4 or BN- $\infty$, inferences on marginal error (co)variance components using the BSt and BN fitted models were similar, found to be sharp and seemingly unbiased, and true parameter values were covered by $95 \%$ equal-tailed PPI of parameters (Table 6).

Average correlations between true and estimated sire effects and average PEV from two replicates using BSt and BN fitted models are presented in Table 7 and 8. When the true model was BSt, both the correlation and PEV indicate that the heavy-tailed fitted models were superior, especially when the true value of $v=4$. When the true model was $\mathrm{BN}$, all fitted models performed identically. In general, the accuracy and PEV results from BSt and BN models suggest that heavy-tailed fitted models can improve accuracy and PEV when the true model is heavy-tailed, but a robust Bayesian analysis using heavy-tailed models does not deteriorate accuracy and PEV if the true model is normal.

\section{Application to gestation length and birth weight}

Inference on degrees of freedom, variance components and heritabilities

The analyses produced PLL values for BSt and BN models of $-47,006$ and $-48,006$ respectively. The log-scale differences between model PLL values for BSt versus BN models were 1,000, which greatly exceeds 2.5 and

Table 4 Average posterior inference on sire (co)variances from ten replicates using the bivariate Student's-t (BSt) and normal (BN) fitted models with different residual degrees of freedom (DF)

\begin{tabular}{|c|c|c|c|c|c|c|c|}
\hline \multirow[b]{3}{*}{ True Parameters } & \multirow[b]{3}{*}{ True Model $^{1}$} & \multicolumn{6}{|c|}{${\text { Fitted } \text { Model }^{2}}^{2}$} \\
\hline & & \multicolumn{3}{|c|}{ BSt } & \multicolumn{3}{|c|}{ BN } \\
\hline & & $\mathrm{PM} \pm \mathrm{SE}^{3}$ & $95 \% \mathrm{PPI}^{4}$ & $\mathrm{ESS}^{5}$ & $\mathrm{PM} \pm \mathrm{SE}$ & $95 \%$ PPI & ESS \\
\hline \multirow[t]{3}{*}{$\overline{\sigma_{s_{1}}^{2}}=2.0$} & BSt-4 & $2.24 \pm 0.16$ & {$[1.32,3.62]$} & 20,983 & $2.31 \pm 0.18$ & {$[1.30,3.83]$} & 20,694 \\
\hline & BSt-12 & $2.44 \pm 0.14$ & {$[1.48,3.88]$} & 24,767 & $2.39 \pm 0.14$ & {$[1.44,3.81]$} & 25,794 \\
\hline & $\mathrm{BN}-\infty$ & $2.49 \pm 0.17$ & {$[1.52,3.93]$} & 26,715 & $2.49 \pm 0.17$ & {$[1.53,3.93]$} & 28,154 \\
\hline \multirow{3}{*}{$\sigma_{s_{1} s_{2}}=1.5$} & BSt-4 & $1.41 \pm 0.17$ & {$[0.40,2.76]$} & 23,326 & $1.43 \pm 0.18$ & {$[0.33,2.89]$} & 23,146 \\
\hline & BSt-12 & $1.93 \pm 0.19$ & {$[0.83,3.47]$} & 27,428 & $1.89 \pm 0.19$ & {$[0.80,3.42]$} & 28,683 \\
\hline & $\mathrm{BN}-\infty$ & $1.77 \pm 0.22$ & {$[0.72,3.23]$} & 28,441 & $1.77 \pm 0.21$ & {$[0.71,3.22]$} & 30,064 \\
\hline \multirow{3}{*}{$\sigma_{s_{2}}^{2}=4.0$} & BSt-4 & $4.23 \pm 0.30$ & {$[2.59,6.70]$} & 24,192 & $4.33 \pm 0.33$ & {$[2.57,6.99]$} & 23,978 \\
\hline & BSt-12 & $4.77 \pm 0.29$ & {$[2.97,7.48]$} & 27,674 & $4.80 \pm 0.30$ & {$[2.98,7.55]$} & 29,102 \\
\hline & $\mathrm{BN}-\infty$ & $4.46 \pm 0.39$ & {$[2.79,6.96]$} & 29,013 & $4.45 \pm 0.39$ & {$[2.78,6.98]$} & 29,365 \\
\hline
\end{tabular}

\footnotetext{
'Used to simulate data

${ }^{2}$ Used in analysis of simulated data

${ }^{3}$ Posterior mean \pm Standard Error

${ }^{4} 95 \%$ equal-tailed posterior probability interval based on the $2.5^{\text {th }}$ and $97.5^{\text {th }}$ percentiles of the posterior density

${ }^{5}$ Effective sample size
} 
Table 5 Average posterior inference on herd variances from ten replicates using the bivariate Student's-t (BSt) and normal (BN) fitted models with different residual degrees of freedom (DF)

\begin{tabular}{|c|c|c|c|c|c|c|c|}
\hline \multirow[b]{3}{*}{ True Parameters } & \multirow[b]{3}{*}{ True Model $^{1}$} & \multicolumn{6}{|c|}{ Fitted Model $^{2}$} \\
\hline & & \multicolumn{3}{|c|}{ BSt } & \multicolumn{3}{|c|}{$\mathrm{BN}$} \\
\hline & & $\mathrm{PM} \pm \mathrm{SE}^{3}$ & $95 \% \mathrm{PPI}^{4}$ & $\mathrm{ESS}^{5}$ & $\mathrm{PM} \pm \mathrm{SE}$ & $95 \%$ PPI & ESS \\
\hline \multirow[t]{3}{*}{$\overline{\sigma_{h_{1}}^{2}}=1.5$} & BSt-4 & $1.71 \pm 0.10$ & {$[1.07,2.56]$} & 12,027 & $1.74 \pm 0.14$ & {$[1.01,2.71]$} & 10,437 \\
\hline & BSt-12 & $1.82 \pm 0.12$ & {$[1.19,2.65]$} & 16,133 & $1.82 \pm 0.12$ & {$[1.18,2.65]$} & 16,802 \\
\hline & $\mathrm{BN}-\infty$ & $1.71 \pm 0.08$ & {$[1.12,2.48]$} & 17,543 & $1.70 \pm 0.08$ & {$[1.12,2.47]$} & 17,537 \\
\hline \multirow[t]{3}{*}{$\sigma_{h_{2}}^{2}=6.0$} & BSt-4 & $6.33 \pm 0.30$ & {$[4.47,8.79]$} & 24,120 & $6.49 \pm 0.28$ & {$[4.47,9.17]$} & 22,810 \\
\hline & BSt-12 & $6.69 \pm 0.28$ & {$[4.77,9.22]$} & 27,704 & $6.72 \pm 0.24$ & {$[4.79,9.28]$} & 27,956 \\
\hline & $\mathrm{BN}-\infty$ & $6.33 \pm 0.27$ & {$[4.55,8.71]$} & 29,800 & $6.33 \pm 0.27$ & {$[4.55,8.71]$} & 29,881 \\
\hline
\end{tabular}

'Used to simulate data

${ }^{2}$ Used in analysis of simulated data

${ }^{3}$ Posterior mean \pm Standard Error

${ }^{4} 95 \%$ equal-tailed posterior probability interval based on the $2.5^{\text {th }}$ and $97.5^{\text {th }}$ percentiles of the posterior density

${ }^{5}$ Effective sample size

decisively indicates the inadequacy of the normality assumption for the distribution of error terms. These results are in agreement with Chang et al. [8] and Cardoso et al. [17], who found that the Student's- $t$ distribution was a better fit to the clinical mastitis data and postweaning gain data, respectively, compared to Slash and normal distributions.

The estimated ESS for $v$ is 1,227 and those for variance components are given in Tables 9, 10 and 11. The ESS for these parameters ranged from 323 to 15,789 , indicating sufficient MCMC mixing. These values were found to be considerably higher than 100, which has been suggested as the minimum ESS for reliable statistical inference [24,25].

The posterior distribution of $v$ from the BSt model, and its posterior mean (M) and 95\% PPI corresponding to the $2.5(\mathrm{~L})$ and $97.5(\mathrm{U})$ percentiles of the posterior distribution are in Figure 1. The posterior mean of $v$ for the BSt model was 3.70 , with $95 \%$ PPI of $(3.44,3.97)$. This density, characterized by small values of $v$ for BSt model confirms that the assumption of normally distributed residuals is not adequate for the analysis of Piemontese GL and BW data.

Posterior inferences on sire-MGS and HYS (co)variances for GL and BW are summarized in Tables 9 and 10 , using posterior means and 95\% PPIs from BSt and $\mathrm{BN}$ models. Posterior distributions of (co)variances were nearly symmetric in BSt and BN models. Posterior means of sire-MGS (co)variances were similar across models, and 95\% PPI widely overlapped. Posterior means of sire-MGS (co)variances from BN model, however, were lower than that from BSt model for GL, and were larger than that from BSt model for BW. Covariances from BN model, including sire or MGS effect for

Table 6 Average posterior inference on marginal error (co)variances from ten replicates using the bivariate Student's$t$ (BSt) and normal (BN) fitted models with different residual degrees of freedom (DF)

\begin{tabular}{|c|c|c|c|c|c|c|c|}
\hline \multirow[b]{3}{*}{ True Parameters } & \multirow[b]{3}{*}{ True Model $^{1}$} & \multicolumn{6}{|c|}{ Fitted Model $^{2}$} \\
\hline & & \multicolumn{3}{|c|}{ BSt } & \multicolumn{3}{|c|}{$\mathrm{BN}$} \\
\hline & & $\mathrm{PM} \pm \mathrm{SE}^{3}$ & $95 \% \mathrm{PPI}^{4}$ & $\mathrm{ESS}^{5}$ & $\mathrm{PM} \pm \mathrm{SE}$ & $95 \%$ PPI & ESS \\
\hline$\overline{\sigma_{E_{1}}^{2}}=30.0$ & BSt-4 & $30.45 \pm 0.51$ & {$[27.44,34.05]$} & 3,336 & $30.29 \pm 0.44$ & {$[28.61,32.07]$} & 42,430 \\
\hline$\sigma_{E_{1}}^{2}=18.0$ & BSt-12 & $17.87 \pm 0.17$ & {$[16.75,19.07]$} & 9,516 & $17.82 \pm 0.16$ & {$[16.83,18.87]$} & 43,135 \\
\hline$\sigma_{E_{1}}^{2}=15.0$ & $\mathrm{BN}-\infty$ & $14.94 \pm 0.11$ & {$[14.10,15.82]$} & 43,387 & $14.94 \pm 0.11$ & {$[14.11,15.82]$} & 43,204 \\
\hline$\sigma_{E_{1} E_{2}}=8.0$ & BSt-4 & $8.20 \pm 0.38$ & {$[6.45,10.10]$} & 11,384 & $8.37 \pm 0.66$ & {$[6.95,9.83]$} & 44,553 \\
\hline$\sigma_{E_{1} E_{2}}=4.8$ & BSt-12 & $4.61 \pm 0.13$ & {$[3.69,5.56]$} & 32,416 & $4.57 \pm 0.12$ & {$[3.72,5.44]$} & 44,370 \\
\hline$\sigma_{E_{1} E_{2}}=4.0$ & $\mathrm{BN}-\infty$ & $3.94 \pm 0.10$ & {$[3.23,4.67]$} & 43,729 & $3.94 \pm 0.10$ & {$[3.23,4.66]$} & 44,238 \\
\hline$\sigma_{E_{2}}^{2}=40.0$ & BSt-4 & $40.07 \pm 0.65$ & {$[35.98,44.94]$} & 3,566 & $39.57 \pm 0.74$ & {$[37.37,41.90]$} & 45,168 \\
\hline$\sigma_{E_{2}}^{2}=24.0$ & BSt-12 & $24.60 \pm 0.31$ & {$[23.04,26.27]$} & 10,145 & $24.54 \pm 0.28$ & {$[23.17,25.98]$} & 45,130 \\
\hline$\sigma_{E_{2}}^{2}=20.0$ & $\mathrm{BN}-\infty$ & $20.13 \pm 0.18$ & {$[19.00,21.31]$} & 42,782 & $20.12 \pm 0.18$ & {$[19.00,21.31]$} & 45,079 \\
\hline
\end{tabular}

'Used to simulate data

${ }^{2}$ Used in analysis of simulated data

${ }^{3}$ Posterior mean \pm Standard Error

${ }^{4} 95 \%$ equal-tailed posterior probability interval based on the $2.5^{\text {th }}$ and $97.5^{\text {th }}$ percentiles of the posterior density

${ }^{5}$ Effective sample size 
Table 7 Average correlations between true and predicted sire effects from ten replicates using the bivariate Student's-t (BSt) and normal (BN) fitted models with different residual degrees of freedom (DF)

\begin{tabular}{lcccc}
\hline & \multicolumn{4}{c}{${\text { Fitted } \text { Model }^{2}}$} \\
\cline { 2 - 5 } True Model'-DF & \multicolumn{2}{c}{ Trait1 } & \multicolumn{2}{c}{ Trait2 } \\
\cline { 2 - 5 } BSt & BN & BSt & BN \\
\hline BSt-4 & 0.90 & 0.87 & 0.92 & 0.90 \\
BSt-12 & 0.93 & 0.93 & 0.95 & 0.95 \\
BN- $\infty$ & 0.94 & 0.94 & 0.95 & 0.95 \\
\hline
\end{tabular}

'Used to simulate data

${ }^{2}$ Used in analysis of simulated data

BW with sire or MGS effect for GL were higher than those from BSt and BS models. Posterior means of HYS variances from BSt and BN models were similar and ranged from 4 to 4.25 for $\mathrm{GL}$, and 2.43 to 2.56 for $\mathrm{BW}$ from the two models. Posterior inference for the marginal residual (co)variances based on $\mathrm{BS} t$ and $\mathrm{BN}$ models are presented in Table 11. The marginal residual variance for $\mathrm{GL}$, and covariance between $\mathrm{GL}$ and $\mathrm{BW}$ from BSt model seemed to agree with those from the $\mathrm{BN}$ model; however, the posterior mean of marginal residual variance for BW from the BSt model was significantly higher than that of the BN model.

Posterior densities of direct and maternal heritabilities, and genetic correlations from BSt and BN models for GL and BW are shown in Figures 2 and 3. Posterior means of direct (0.47) and maternal (0.29) heritabilities from BSt and BN models were similar for GL. However, posterior means of direct (0.28) and maternal (0.23) heritabilities from $\mathrm{BN}$ models were higher than those $(0.23$ and 0.18) from the heavy-tailed model for BW (Figure 2). In contrast to our findings, Cardoso et al. [7] and Chang et al. [8] have found no real difference in posterior means for heritabilities whether using Student's- $t$, Slash or normal models. Posterior means of direct heritabilities from BSt and BN models for GL and BW traits were lower; however, those of maternal heritabilities were higher than the values reported by Ibi et al. [26] and Crews [27]. Posterior means $(-0.87,-0.86)$ of genetic

Table 8 Prediction error variance of sire effects using the bivariate Student's-t (BSt) and normal (BN) fitted models with different residual degrees of freedom (DF)

\begin{tabular}{|c|c|c|c|c|}
\hline \multirow[b]{3}{*}{ True Model'-DF } & \multicolumn{4}{|c|}{ Fitted Model $^{2}$} \\
\hline & \multicolumn{2}{|c|}{ Trait1 } & \multicolumn{2}{|c|}{ Trait2 } \\
\hline & BSt & BN & BSt & $\mathrm{BN}$ \\
\hline$\overline{\mathrm{BSt}-4}$ & 0.36 & 0.44 & 0.51 & 0.67 \\
\hline BSt-12 & 0.29 & 0.30 & 0.41 & 0.44 \\
\hline $\mathrm{BN}-\infty$ & 0.23 & 0.23 & 0.33 & 0.33 \\
\hline
\end{tabular}

${ }^{1}$ Used to simulate data

${ }^{2}$ Used in analysis of simulated data
Table 9 Posterior inference on sire-MGS (co)variances for gestation length (GL) and birth weight (BW) using the bivariate Student's-t (BSt) and normal (BN) models

\begin{tabular}{lcccccc}
\hline & \multicolumn{3}{c}{$\mathbf{B S t}$} & \multicolumn{4}{c}{ BN } \\
\cline { 2 - 7 } Parameters & $\mathbf{P M}^{\mathbf{1}}$ & $\mathbf{9 5 \% ~ \text { PPI } ^ { \mathbf { 2 } }}$ & $\mathbf{E S S}^{\mathbf{3}}$ & $\mathbf{P M}$ & $\mathbf{9 5 \%}$ PPI & ESS \\
\hline$\sigma_{s_{G L}}^{2}$ & 8.42 & {$[6.65,10.43]$} & 894 & 8.13 & {$[6.27,10.31]$} & 384 \\
$\sigma_{s_{G L} s_{B W}}$ & 0.13 & {$[-0.43,0.72]$} & 774 & 0.16 & {$[-0.48,0.81]$} & 496 \\
$\sigma_{s_{G L} m_{G L}}$ & 2.75 & {$[1.77,3.76]$} & 567 & 2.73 & {$[1.63,3.81]$} & 323 \\
$\sigma_{s_{G L} m_{B W}}$ & -0.54 & {$[-1.04,-0.04]$} & 524 & -0.74 & {$[-1.32,-0.21]$} & 405 \\
$\sigma_{s_{B W}}^{2}$ & 1.02 & {$[0.68,1.43]$} & 528 & 1.12 & {$[0.75,1.55]$} & 550 \\
$\sigma_{s_{B W} m_{G L}}^{2}$ & 0.26 & {$[-0.13,0.69]$} & 429 & 0.40 & {$[-0.09,0.90]$} & 230 \\
$\sigma_{s_{B W} m_{B W}}$ & 0.36 & {$[0.15,0.58]$} & 428 & 0.39 & {$[0.18,0.62]$} & 484 \\
$\sigma_{m_{G L}}^{2}$ & 2.24 & {$[1.47,3.16]$} & 389 & 2.04 & {$[1.17,3.05]$} & 232 \\
$\sigma_{m_{G L} m_{B W}}$ & 0.27 & {$[-0.03,0.57]$} & 430 & 0.32 & {$[-0.01,0.69]$} & 336 \\
$\sigma_{m_{B W}}^{2}$ & 0.53 & {$[0.34,0.74]$} & 457 & 0.59 & {$[0.38,0.87]$} & 371 \\
\hline
\end{tabular}

${ }^{1}$ Posterior mean

${ }^{2} 95 \%$ equal-tailed posterior probability interval based on the $2.5^{\text {th }}$ and $97.5^{\text {th }}$ percentiles of the posterior density

${ }^{3}$ Effective sample size

correlations between D and M effects of GL, and those $(-0.73,-0.71)$ of BW from BSt and BN models in Figure 3 were significantly negative and very similar with overlapping posterior densities. They were higher than those reported in literature [26,27], and the negative posterior mean of the genetic correlation implies an antagonistic relationship between $\mathrm{D}$ and $\mathrm{M}$ effects. The posterior

Table 10 Posterior inference on herd-year-season (co) variances for gestation length (GL) and birth weight (BW) using the bivariate Student's-t (BSt) and normal (BN) models

\begin{tabular}{lcccccc}
\hline & \multicolumn{3}{c}{ BSt } & \multicolumn{3}{c}{ BN } \\
\cline { 2 - 7 } Parameters & $\mathbf{P M}^{\mathbf{1}}$ & $\mathbf{9 5 \%} \mathbf{P P I}^{\mathbf{2}}$ & $\mathbf{E S S}^{\mathbf{3}}$ & $\mathbf{P M}$ & $\mathbf{9 5 \%}$ PPI & ESS \\
\hline$\sigma_{h_{G L}^{2}}^{2}$ & 4.00 & {$[3.02,5.10]$} & 2,122 & 4.21 & {$[3.00,5.60]$} & 1,661 \\
$\sigma_{h_{B W}}^{2}$ & 2.43 & {$[2.04,2.85]$} & 3,282 & 2.56 & {$[2.14,3.00]$} & 3,403 \\
\hline
\end{tabular}

${ }^{1}$ Posterior mean

${ }^{2} 95 \%$ equal-tailed posterior probability interval based on the $2.5^{\text {th }}$ and $97.5^{\text {th }}$ percentiles of the posterior density

${ }^{3}$ Effective sample size

Table 11 Posterior inference on marginal residual (co) variances for gestation length (GL) and birth weight (BW) using the bivariate Student's-t (BSt) and normal (BN) models

\begin{tabular}{|c|c|c|c|c|c|c|}
\hline \multirow[b]{2}{*}{ Parameters } & \multicolumn{3}{|c|}{ BSt } & \multicolumn{3}{|c|}{$\mathrm{BN}$} \\
\hline & $\mathrm{PM}^{1}$ & $95 \% \mathrm{PPI}^{2}$ & $\mathrm{ESS}^{3}$ & PM & 95\% PPI & ESS \\
\hline$\overline{\sigma_{E_{G L}}^{2}}$ & 51.86 & {$[48.43,55.84]$} & 2,376 & 48.90 & {$[47.22,50.64]$} & 9,039 \\
\hline$\sigma_{E_{G L} E_{B W}}$ & 3.77 & {$[3.01,4.56]$} & 8,213 & 3.20 & {$[2.63,3.78]$} & 11,671 \\
\hline$\sigma_{E_{B W}}^{2}$ & 13.37 & {$[12.47,14.41]$} & 2,367 & 11.14 & {$[10.76,11.53]$} & 15,789 \\
\hline
\end{tabular}




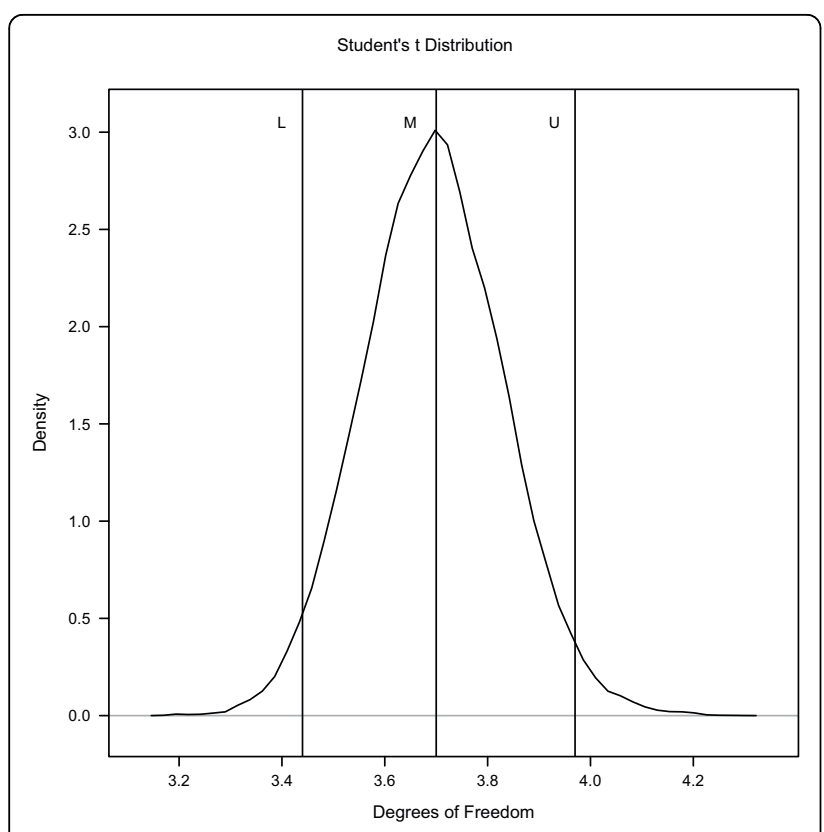

Figure 1 Posterior densities of degrees of freedom obtained from bivariate Student's- $t$ (BSt) model fitted to gestation length (GL) and birthweight (BW). M represents posterior mean, $L$ represents the $2.5^{\text {th }}$ percentiles of the posterior density, $\mathrm{U}$ represent $97.5^{\text {th }}$ percentiles of the posterior density. densities of genetic correlations between $\mathrm{D}$ effects on one trait and $\mathrm{M}$ effects on another included zero, indicating non-significant correlations.

The posterior means of $\lambda_{i}$ in the BSt model can be used to assess the extent to which any particular pair of records presents an outlier for either trait in comparison to a normal error assumption. Low values of $\lambda_{i}$ (i.e. closer to zero) indicate at least one deviant record among the two traits, whereas values of $\lambda_{i}$ close to 1 show that the corresponding pair of records match the normal model [17]. The ranges of posterior means of $\lambda_{i}$ obtained for different animals from the BSt models varied between 0.09 and 1.75. The values of $\lambda_{i}$ are plotted against estimated values of residuals for BW and GL in Figure 4. The distributions of posterior means of $\lambda_{i}$ less than 0.3 (left figure) or less than 0.2 (right figure) are given in Figure 4 . The figure on the right plots posterior mean values of $\lambda_{i}$ less than 0.2 , representing outliers 3 or more standard deviations (SD) from the mean for GL or BW. When the posterior mean values of $\lambda_{i}$ are close to unity, the estimated values of residuals approach normally distributed residuals, indicating adequate model fit.

In general, random effects contributing to bivariate traits may be correlated positively, negatively or uncorrelated. Accordingly, it is reasonable that effects may
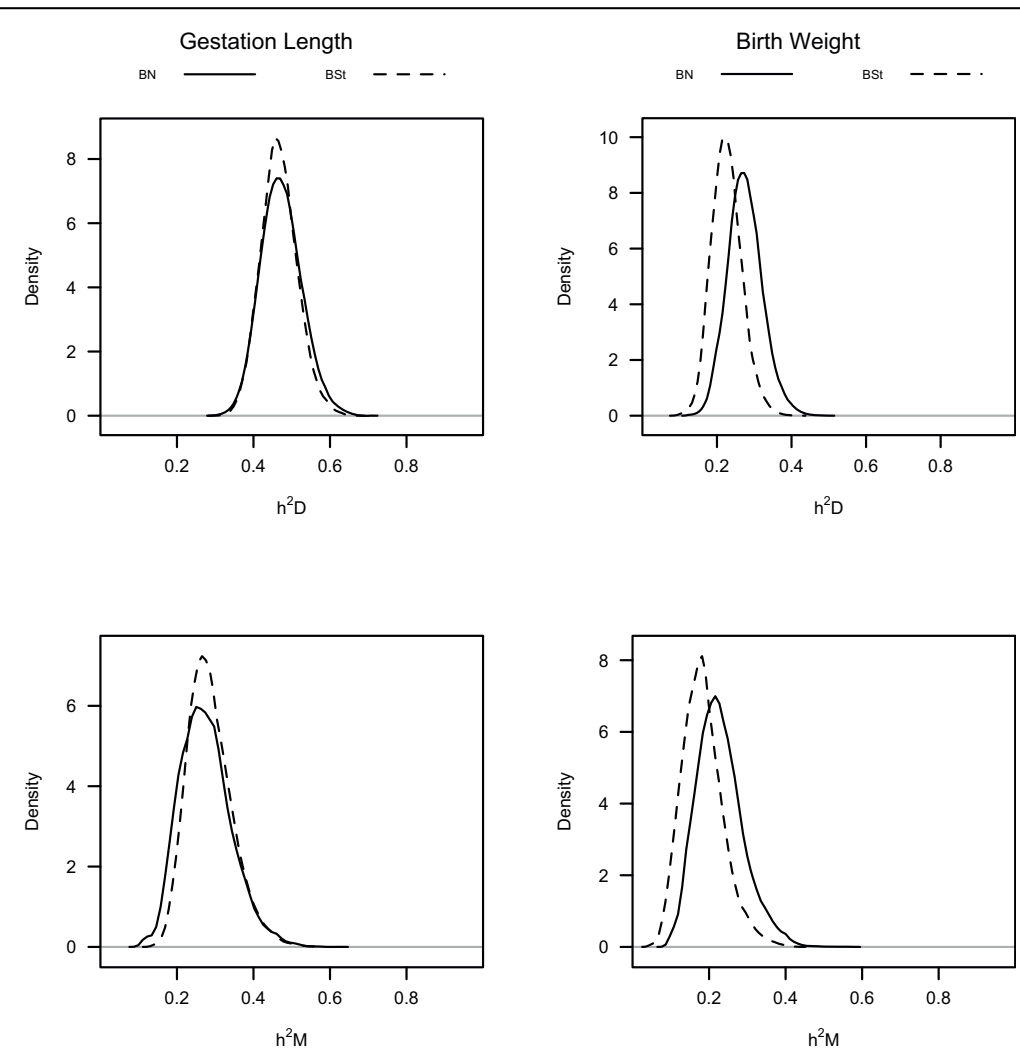

Figure 2 Posterior densities of direct (D) and maternal (M) heritabilities of gestation length (GL) and birth weight (BW) obtained from bivariate Student's-t (BSt) or normal (BN) models. $h^{2} D$ and $h^{2} M$ represent direct and maternal heritabilities. 

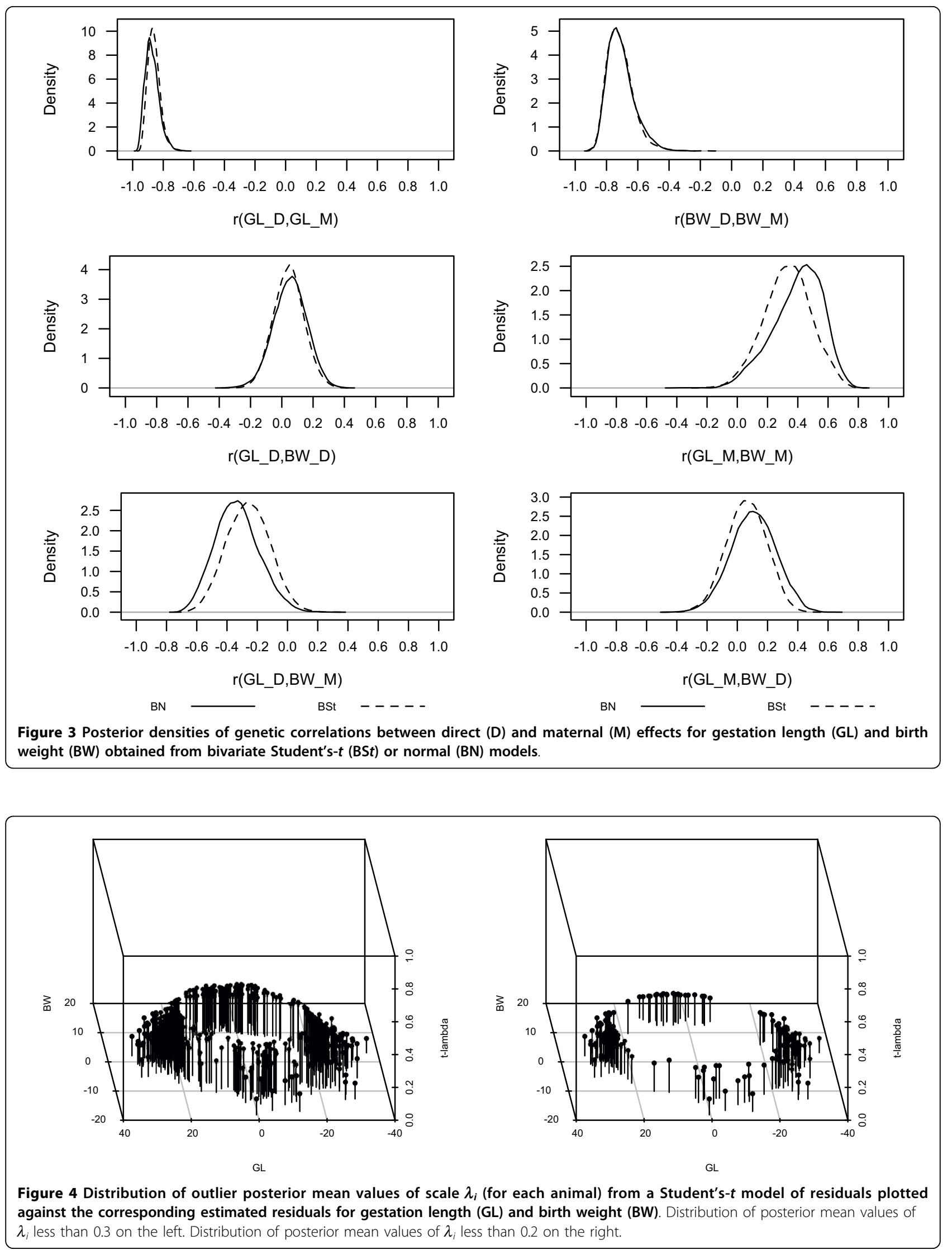
vary in their distribution and that residuals for one trait might conceptually be heavy-tailed while others may be light-tailed. Further, it is conceivable that individual animals could exhibit trait specific lambda values. However, in the context of the traits considered in this experiment, it is not unreasonable to imagine that the lambda values could be consistent across the traits because gestation length and birth weight are positively correlated, at phenotypic, genetic and residual levels, and that non-genetic effects that produce residual outliers for one trait such as gestation length might similarly effect birth weight. In fact, single-trait analyses of GL and BW indicate that both traits are heavy-tailed with 2.91 (GL) and 3.66 (BW) for posterior means of $v$. A more general model that assumes a bivariate distribution for trait-specific $\lambda$ values is more technically demanding than the model used in this paper, but warrants further research.

\section{Inference on sire effects}

Sire ranking based on posterior means of the sire effects from BSt and BN models for GL and BW compared using Kendall rank correlations are in Figure 5. The rank correlation between $\mathrm{BN}$ and $\mathrm{BSt}$ models was 0.77 for GL, and 0.81 for BW, indicating re-ranking of sires among models.

Considering only sires ranked in the top 100 for GL and $\mathrm{BW}$ using the $\mathrm{BN}$ model, $82 \%$ and $75 \%$ of them were found to be same for GL and BW in the top 100 animals by BSt model. The rank correlations between $\mathrm{BN}$ and BSt models decreased considerably to about 0.6 for GL and about 0.5 for BW (Figure 5). Cardoso et al. [17] have found similar results in a multibreed genetic evaluation of postweaning gain in Nelore-Hereford cattle and have suggested that a low rank correlation among the top sires may have greater implications for genetic evaluations and selection decisions than the correlation results involving all sires. Figure 5 shows that posterior means of sire effects from BN model shrank to a greater extent under the BSt model. Substantial re-ranking of sires was observed due to the greater shrinkage of the posterior mean of sire effects in BSt model, and this reranking was more pronounced in BW than in GL. Stranden and Gianola [28] have pointed out that

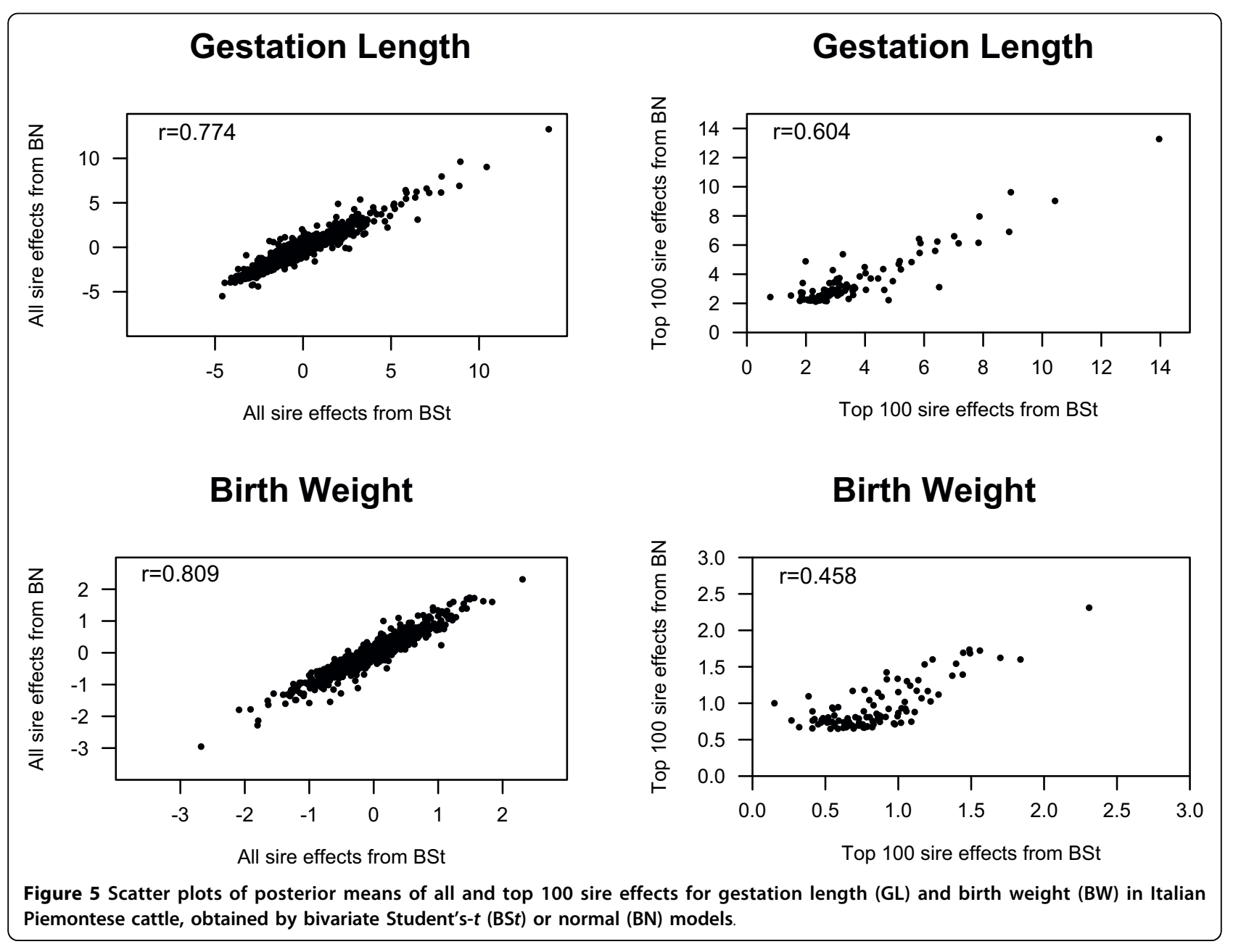


animals that are phenotypic outliers will exhibit more extreme predictions of genetic merit under the BN model compared to the heavy-tailed models that mute the effects of the large residuals.

\section{Conclusions}

Bayesian techniques are capable of fitting models where residuals have a heavy-tailed distribution with unknown degrees of freedom. Model comparisons, using PLL, in the simulation study typically favoured the BSt models over the $\mathrm{BN}-\infty$ model when the true models were heavy-tailed. Further, there was no difference in PLL between $\mathrm{BS} t$ and $\mathrm{BN}-\infty$ models and there were no disadvantages of fitting a BSt model when the true model was normal.

Bivariate residual distributions can be assumed normal, or Student's- $t$ in the analysis of field data. Predictive log-likelihood values used as model choice criteria in the bivariate analysis of GL and BW data indicated that the BSt model with low degrees of freedom fitted better than the BN model. Posterior means of direct and maternal heritabilities from the BN model were similar or higher than those from the BSt model. Appreciable differences were observed in sire ranking overall and specifically in the top 100 sires based on rank correlations between BSt and BN model sire effects. These results indicate that genetic evaluation and selection strategies will be sensitive to the assumed model. Animals whose $\lambda_{i}$ values were close to zero in BSt model were identified as having one or more outlying records. An interesting extension for future studies would be that of allowing different scale parameter specification for each trait in the BSt model.

\section{Acknowledgements}

This project was supported by grant TUBITAK TOVAG-1070915 from the Scientific and Technological Research Council of Turkey (Project coordinator: Dr. Kadir KIZILKAYA). ANABORAPI (Associazione nazionale alleatori bovini di razza Piemontese, Strada Trinitá 32a, 12061 Carrú, Italy) is gratefully acknowledged for providing the data for this study. We are grateful to I. Misztal for making available Sparsem90 and Fspak90.

\section{Author details}

${ }^{1}$ Department of Animal Science, lowa State University, Ames, IA 50011 USA. 2Department of Animal Science, Adnan Menderes University, Aydin 09100 Turkey. ${ }^{3}$ Institute of Veterinary, Animal and Biomedical Sciences, Massey University, Palmerston North, New Zealand. ${ }^{4}$ Department of Animal Science, University of Ankara, Diskapi Ankara 06110 Turkey.

\section{Authors' contributions}

KK carried out the simulation and data analysis and drafted the manuscript DJG and RLF provided support for statistical analysis in the study and helped to draft the manuscript. BM participated in the design of the study and statistical analysis. MAY helped to design and coordinate the study. All authors read and approved the final manuscript.

\section{Competing interests}

The authors declare that they have no competing interests.
Received: 19 January 2010 Accepted: 30 June 2010

Published: 30 June 2010

\section{References}

1. Roger WH, W TJ: Understanding some long-tailed distributions. Statistica Neerlandia 1972, 26:211 226.

2. Lange KL, Little RJA, Taylor JMG: Robust Statistical Modeling Using the t Distribution. J Am Stat Assoc 1989, 84:881-896.

3. Kizilkaya K, Carnier P, Albera A, Bittante G, Tempelman R: Cumulative t-link threshold models for the genetic analysis of calving ease scores. Genet Sel Evol 2003, 35:489-512.

4. Stranden I, Gianola D: Mixed effects linear models with t-distributions for quantitative genetic analysis: a Bayesian approach. Genet Sel Evol 1999, 31:25-42.

5. vonRohr P, Hoeschele I: Bayesian QTL mapping using skewed Student-t distributions. Genet Sel Evol 2002, 34:1-21.

6. Rosa GJM, Padovani CR, Gianola D: Robust linear mixed models with normal/independent distributions and Bayesian MCMC implementation. Biom J 2003, 45:573-590.

7. Cardoso FF, Rosa GJM, Tempelman RJ: Multiple-breed genetic inference using heavy-tailed structural models for heterogeneous residual variances. J Anim Sci 2005, 83:1766-1779.

8. Chang YM, Andersen-Ranberg IM, Heringstad B, Gianola D, Klemetsdal G: Bivariate analysis of number of services to conception and days open in Norwegian red using a censored threshold-linear model. J Dairy Sci 2006, 89:772-778.

9. Sorensen DA, Gianola D: Likelihood, Bayesian and MCMC methods in Quantitative Genetics New York: Springer-Verlag, New York, Inc 2002.

10. Searle SR: Matrix Algebra Useful for Statistics New York: John Wiley \& Sons 1982.

11. Chib S, Greenberg E: Understanding the Metropolis-Hastings Algorithm. Am Stat 1995, 49:327-335.

12. Geman D, Geman S: Stochastic relaxation, Gibbs distributions, and the Bayesian restoration of images. IEEE Trans Pattern Anal Mach Intell 1984, 6:721-741.

13. Gelfand AE, Smith AFM: Sampling-Based Approaches to Calculating Marginal Densities. J Am Stat Assoc 1990, 85:398-409.

14. Kizilkaya K, Tempelman R: A general approach to mixed effects modeling of residual variances in generalized linear mixed models. Genet Sel Evol 2005, 37:31-56.

15. Carnier P, Albera A, Dal Zotto R, Groen AF, Bona M, Bittante G: Genetic parameters for direct and maternal calving ability over parities in Piedmontese cattle. J Anim Sci 2000, 78:2532-2539.

16. Luo MF, Boettcher PJ, Schaeffer LR, Dekkers JC: Bayesian inference for categorical traits with an application to variance component estimation. J Dairy Sci 2001, 84:694-704.

17. Cardoso FF, Rosa GJM, Tempelman RJ: Accounting for outliers and heteroskedasticity in multibreed genetic evaluations of postweaning gain of Nelore-Hereford cattle. J Anim Sci 2007, 85:909-918.

18. Gelfand AE: Model determination using sampling-based methods. Markov Chain Monte Carlo in Practice London, UK: Chapman and HallGilks WR, Richardson S, Spiegelhalter DJ 1996, 145-161.

19. Raftery AE: Hypothesis testing and model selection. Markov Chain Monte Carlo in Practice London, UK: Chapman and HallGilks WR, Richardson S, Spiegelhalter DJ 1996, 163-187.

20. Heidelberger $P$, Welch PD: Simulation run length control in the presence of an initial transient. Oper Res 1983, 31:1109-1144.

21. Plummer M, Best N, Cowles K, Vines K: CODA: Convergence Diagnosis and Output Analysis for MCMC. R News 2006, 6[http://CRAN.R-project.org/ doc/Rnews/].

22. Geyer CJ: Practical Markov chain Monte-Carlo (with discussion). Stat Sci 1992, 7:467-511.

23. Sorensen DA, Andersen S, Gianola D, Korsgaard I: Bayesian inference in threshold models using Gibbs sampling. Genet Sel Evol 1995, 27:229-249.

24. Bink MCAM, Quaas RL, Van Arendonk JAM: Bayesian estimation of dispersion parameters with a reduced animal model including polygenic and QTL effects. Genet Sel Evol 1998, 30:103-125.

25. Uimari $P$, Thaller $G$, Hoeschele I: The use of multiple markers in a Bayesian method for mapping quantitative trait loci. Genetics 1996, 143:1831-1842. 
26. Ibi T, Kahi AK, Hirooka H: Genetic parameters for gestation length and the relationship with birth weight and carcass traits in Japanese Black cattle. Anim Sci J 2008, 79:297-302.

27. Crews DH Jr: Age of dam and sex of calf adjustments and genetic parameters for gestation length in Charolais cattle. J Anim Sci 2006, 84:25-31.

28. Stranden I, Gianola D: Attenuating effects of preferential treatment with Student-t mixed linear models: a simulation study. Genet Sel Evol 1998, 30:565-583.

doi:10.1186/1297-9686-42-26

Cite this article as: Kizilkaya et al:: Use of linear mixed models for genetic evaluation of gestation length and birth weight allowing for heavy-tailed residual effects. Genetics Selection Evolution 2010 42:26.

\section{Submit your next manuscript to BioMed Central} and take full advantage of:

- Convenient online submission

- Thorough peer review

- No space constraints or color figure charges

- Immediate publication on acceptance

- Inclusion in PubMed, CAS, Scopus and Google Scholar

- Research which is freely available for redistribution

Submit your manuscript at www.biomedcentral.com/submit 